\title{
ORT.3 I - CAR-T cells generated using Sleeping Beauty vectors and expan- ded with a lymphoblastoid cell line (LCL) display in vivo antitumor activity.
}

Leonardo Chicaybam ${ }^{1 \star}$; Mayra Carneiro²; Luiza Abdo²; Barbara Peixoto²; Mariana Viegas; Priscila de Sousa ${ }^{2}$; Luciana Carvalho²; Martín Hernán Bonamino ${ }^{1}$.

1Fiocruz - Fundação Oswaldo Cruz;

2INCa.

Introduction: Anti-CD19 CAR-T cell immunotherapy for treatment of cancer is showing promising results in in patients harboring $\mathrm{B}$ cell malignancies, with overall response rates of $73 \%$. However, there is no standard protocol for the generation of CAR-modified T cells, with different genetic modification vectors and expansion protocols being used. Viral vectors insert the transgene in the genome of the cell, providing long term CAR expression. However, their use is associated with high production costs and cumbersome quality controls, impacting the final cost of CAR-T cell therapies. Sleeping Beauty (SB) transposon system consist of plasmidbased integrative vectors that, through a cut-and-paste mechanism catalyzed by a transposase, recognize inverted terminal repeats flanking the transgene and insert it in the target cell genome. We show here that this system, combined with LCL-based T cell expansion can be used to efficiently transfect primary $\mathrm{T}$ lymphocytes and induce long term CAR expression throughout $\mathrm{T}$ cell expansion.

Objective: To develop a protocol to expand SB-transfected CAR T cells using a LCL.

Methodology: Peripheral blood mononuclear cells (PBMCs) were isolated using Ficoll and transfected using Nucleofector II electroporator. Plasmids encoding the anti-CD19 CAR $(19 \mathrm{BBz})$ and transposase (SB100X) used in the transfection were mixed based on a pre-defined optimized proportion. After transfection cells were stimulated with the L388 LCL, a mature $\mathrm{B}$ cell line that expresses the target antigen (CD19) and costimulatory receptors. CAR T cells were re-stimulated up to 3 times with L388 and T cell phenotype (CAR expression, memory markers) and effector function were evaluated at the end of each cycle. In vivo effector activity was evaluated against RS4;11 leukemia cells xenografted in NSG mice.

Results: Electroporation of PBMCs with transposon plasmid decreased viability and altered the frequency of memory subpopulations when compared to the mock (electroporated without plasmid) condition. However, CAR expression rescued the electroporated lymphocytes and these cells showed increased proliferation compared to mock control (28-fold vs 15-fold expansion; $19 \mathrm{BBz}$ vs mock). Moreover, CAR+ lymphocytes showed an increased frequency by the end of the stimulation cycle compared to d1 post electroporation. NK cell depletion prior to L388 stimulation altered the composition of memory subpopulations, favoring the expansion of CD8+ CD62L- CCR7+ cells. CAR-T cells expanded with L388 also showed high antitumor activity in vivo, increasing the overall survival when compared to mock cells.

Conclusion: The results showed that electroporation using the SB system is a simple and costeffective method for inducing long-term CAR expression in T lymphocytes. Expansion of CAR-T cells was possible by using the L388, providing up to 3 cycles of stimulations and reaching the required cell number for preclinical testing. The high in vivo effector activity seen in the RS4;11 leukemia model encourages the translation of this approach to clinical application.

Keywords: chimeric antigen receptor; immunotherapy; T lymphocyte 